\title{
ESCALA DOS QUESTIONÁRIOS PARA ANÁLISE DA EXPERIÊNCIA DO USUÁRIO: ESTUDO PRELIMINAR
}

Ana Renata de Mantova

UNESP/FAAC - PPGDesign

anamantova@hotmail.com

Ana Cristina Maurício Ferreira

UNESP/ FAAC - PPGDesign

anacr.fatec@gmail.com
Paula da Cruz Landim

UNESP/ FAAC - PPGDesign

paula@faac.unesp.br

João Fernando Marar

UNESP/ FAAC - PPGDesign

fermarar@fc.unesp.br

Resumo: O anseio do designer de todos os âmbitos projetuais é suprir as necessidades, desejos e expectativas dos usuários de seus produtos. Através de metodologias abordadas no estudo da experiência do usuário é possível alcançar o potencial e entender como os sentimentos das pessoas são elaborados durante o uso de seus produtos. O questionário e sua respectiva escala de medição é uma das metodologias adotadas para a análise da experiência do usuário e sua adequada escolha pode contribuir para respostas efetivas do usuário e auxiliar na compilação dos dados coletados. Este artigo fornece uma visão geral dos questionários adotados e a análise de suas relativas escalas.

Palavras-chave: Experiência do Usuário, Metodologia, Escala de Medição.

\begin{abstract}
The desire of the designer of all projective areas is to meet the needs, desires and expectations of their users products. Through methodologies covered in the user experience of study it is possible to reach the potential and understand how people's feelings are prepared for the use of their products. The questionnaire and their respective measuring range is one of the methodologies adopted for the analysis of user experience and appropriate choice can contribute to effective responses user and assist in the compilation of the data collected. This article provides an overview of the adopted questionnaires and analysis of their relative scales.
\end{abstract}

Keywords: User Experience, Methodology, Measurement Scale. 


\section{INTRODUÇÃO}

O design é um processo complexo e dinâmico na sua essência, pois trata diretamente com a proposta de soluções dos problemas das pessoas (Lawson, 2006). Neste contexto, o designer precisa saber o potencial daqueles que irão fazer uso de seus projetos (Stappers, 2006). Portanto entender as necessidades e expectativas do usuário é essencial para o design ser bem sucedido.

A experiência do usuário é uma disciplina que auxilia o Design a coletar dados emocionais que auxiliam na projetação. Compreender os sentimentos dos usuários é o foco dos pesquisadores dessa área. Saber se estão se divertindo, frustrados, interessados? Entender como um usuário se sente em reação a uma experiência pode ajudar a aperfeiçoar os aspectos positivos e excluir ou ao menos disfarçar os aspectos negativos de determinado produto. (Schall, 2015).

O termo experiência do usuário (user experience - UX) foi proposto por Don Norman. Em uma entrevista no UX Week 2008, o autor relata que a interface humana e a usabilidade são disciplinas limitadas e por isso quis abranger com somente um tema todos os aspectos da experiência de uma pessoa. (Norman, 2008).

A Associação Brasileira de Normas Técnicas (ABNT), mediante a NBR ISO 9241210:2011 apresenta a definição da experiência do usuário como sendo "percepções e respostas das pessoas, resultantes do uso e/ou uso antecipado de um produto, sistema ou serviço."

Uma maneira comum de analisar a experiência do usuário é através de questionários, no entanto é importante reconhecer que os participantes nem sempre são objetivos. Novos dispositivos como o EyeTracking e a Eletroencefalografia encontram-se no mercado para desmistificar as análises subjetivas. Porém, ainda nenhum método captura completamente a experiência do usuário e as metodologias são complementares ao combinar seus benefícios é possível identificar particularidades com a experiência, ou ainda corroborar os resultados obtidos. (Schall, 2015).

Portanto um questionário com uma escala bem definida é importante para que as análises sejam fáceis de serem interpretadas pelo pesquisador, além de facilitar o preenchimento sem causar cansaço ou confusão do respondente, mantendo assim a fidelidade das informações. Este trabalho propõe um levantamento de métodos com o formato de questionário com escalas e a análise das mesmas.

\section{MÉTODOS DE ANÁLISE DA EXPERIÊNCIA DO USUÁRIO NO FORMATO DE QUESTIONÁRIOS E ESCALA}

Foram relacionados 20 métodos de análise da experiência do usuário no formato de questionários (Vermeeren et al., 2010), abaixo segue a relação dos métodos descritos em inglês e a respectiva sugestão de tradução para o português bem como descrição de cada uma delas:

Aesthetics Scale - Escala Estética: foi desenvolvido por pesquisadoras da Universidade de Negev Israel para mensurar fatores estéticos de sites da web. (Lavie, 2004). A escala utilizada para este método não foi disponibilizada.

Affect Grid - Grade Afetiva: apresenta uma escala projetada como um meio rápido de avaliar afeto ao longo das dimensões do prazer/desprazer e 
excitação/sonolência. Os autores são das Universidades do Canadá e Califórnia. (Russell, 1989).

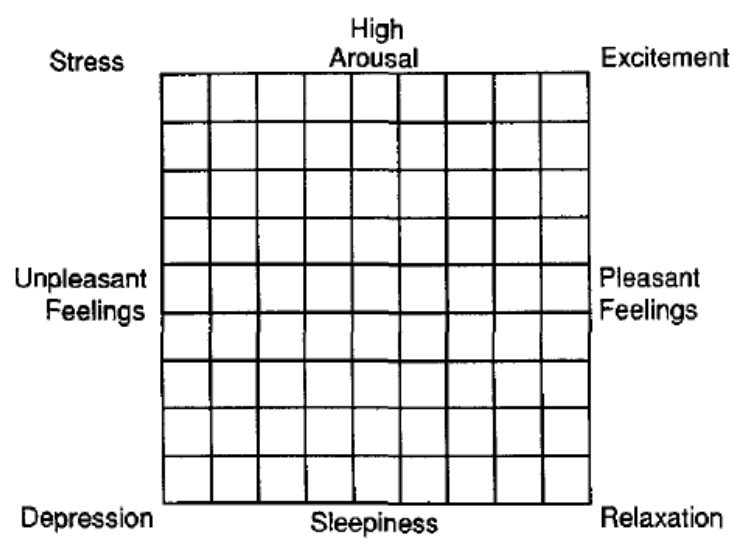

Figura 1: Exemplo da escala Affect Grid.

Fonte: Russell, 1989.

Attrak-Work Questionnaire - sem sugestão para tradução: questionário para avaliação da experiência do usuário no contexto do jornalismo em aparelhos de telefone celular. Desenvolvido por pesquisadores da Finlândia. (Väätäjä, 2009). A escala utilizada para este método não foi disponibilizada.

AttrakDiff - sem sugestão para tradução: Instrumento online para medir a atratividade de um produto interativo através de pares de adjetivos opostos. $O$ sistema foi desenvolvido na Alemanha. (AttrakDiff, 2016).

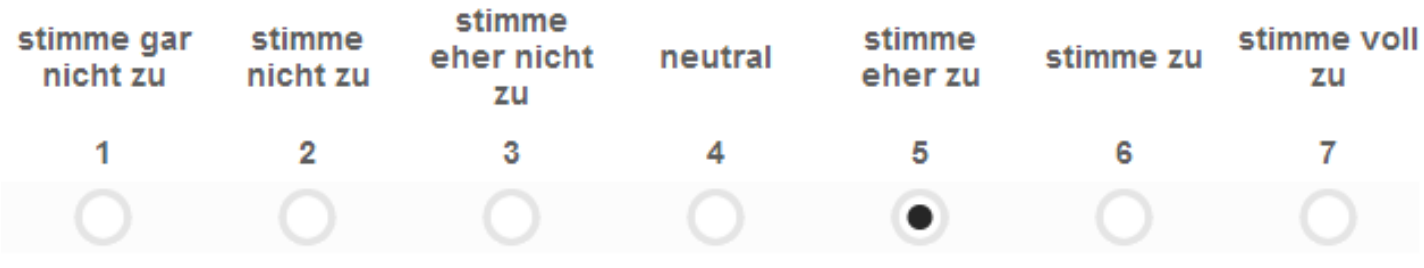

Figura 2: Exemplo da escala AttrakDiff.

Fonte: AttrakDiff, 2016.

Differential Emotions Scale (DES) - Escala de Diferenciais Emocionais: Criado pelo professor estadunidense Carroll Ellis Izard é um instrumento que considera quantas vezes o usuário experimentou determinada emoção durante a experiência. (AllaboutUX, 2016). A escala utilizada para este método não foi disponibilizada.

Game Experience Questionnaire (GEQ) - Questionário para Experiência de Jogos: Desenvolvido por uma equipe interdisciplinar da Universidade de Tecnologia da Holanda, este método acessa a experiência das crianças em jogos digitais. (Game Experience Lab, 2016). A escala utilizada para este método não foi disponibilizada.

Geneva Appraisal Questionnaire (GAQ) - Questionário de Avaliação de Genebra: Desenvolvido pelo Grupo de Pesquisa em Emoção de Genebra, o GAQ tem a finalidade de avaliar um episódio emocional específico. (Affective Sciences, 2002). 
not at all moderately extremely does not apply

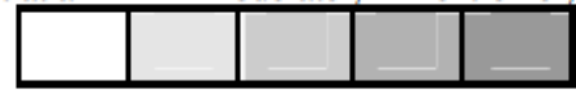

$\mathrm{O}$

Figura 3: Exemplo da escala Geneva Appraisal Questionnaire.

Fonte: Affective Sciences, 2002.

Geneva Emotion Wheel (GEW) - Roda de Emoções de Genebra: Também desenvolvido pelo Grupo de Pesquisa em Emoção de Genebra, é um instrumento teórico para medir reações emocionais a objetos, eventos e situações. (Affective Sciences, 2012).

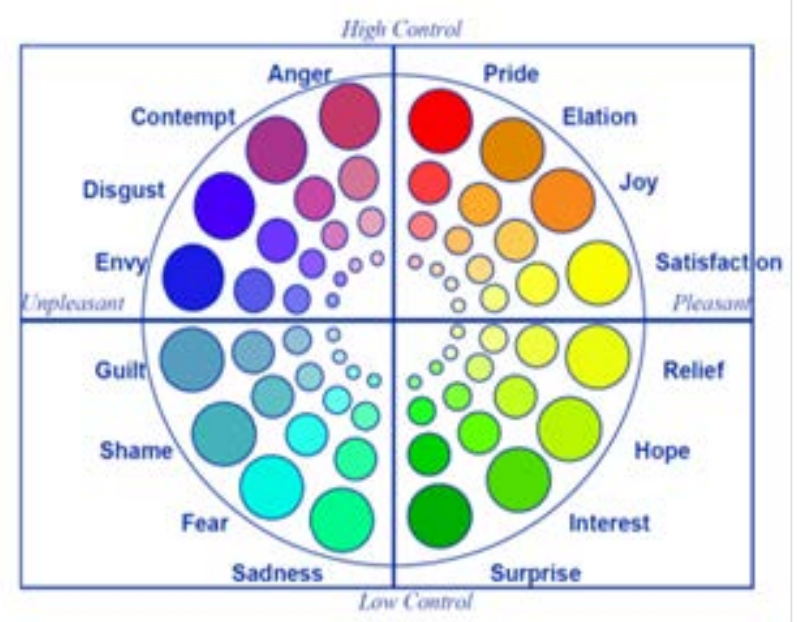

Figura 4: Exemplo da escala Geneva Emotion Wheel. Fonte: Affective Sciences, 2012.

Hedonic Utility Scale (HED/UT) - Escala para abordar aspectos hedônicos e dimensões de utilidade. (VOSS, K. E. et al, 2003). A escala utilizada para este método não foi disponibilizada.

Intrinsic Motivation Inventory (IMI) - Inventário de Motivação Intrínseca: Dispositivo de medição multidimensional destinado a avaliar experiências em laboratório. (Self Determination Theory, 2016).

$\begin{array}{ccccccc}1 & 2 & 3 & 4 & 5 & 6 & 7 \\ \begin{array}{c}\text { not at all } \\ \text { true }\end{array} & & \begin{array}{c}\text { somewhat } \\ \text { true }\end{array} & & & \begin{array}{c}\text { very } \\ \text { true }\end{array}\end{array}$

Figura 5: Exemplo da escala Intrisic Motivation Inventory.

Fonte: Self Determiation Theory, 2016.

Perceived Comfort Assessment - Avaliação do Conforto Percebido: para avaliação do conforto percebido em assentos de carro. (AllaboutUX, 2016). A escala utilizada para este método não foi disponibilizada. 
Positive and Negative Affect Scale (PANAS) - Escala de Afeto: desenvolvida para medir as duas dimensões primárias do humor: o afeto positivo e negativo. (WATSON, D. et al, 1988).

$\begin{array}{ccccc}1 & 2 & 3 & 4 & 5 \\ \begin{array}{l}\text { Very Slightly or Not } \\ \text { at All }\end{array} & \text { A Little } & \text { Moderately } & \text { Quite a Bit } & \text { Extremely }\end{array}$

Figura 6: Exemplo da escala Positive and Negative Affect Scale.

Fonte: (WATSON, D. et al, 1988).

Presence Questionnaire - Questionário de Presença: escala para ambiente virtual (WITMER, B. G.; SINGER, M. J., 1998).

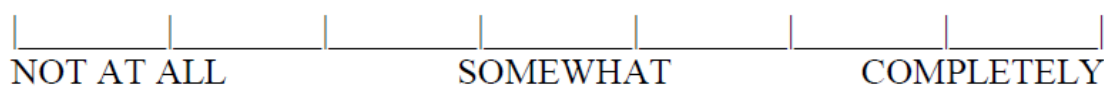

Figura 7: Exemplo da escala Presence Questionnaire.

Fonte: (WITMER, B. G.; SINGER, M. J., 1998).

Product Semantic Analysis (PSA) - Análise Semântica do Produto: a escala semântica é construída para cada caso de avaliação. (AllaboutUX, 2016).

QSA GQM Questionnaires - Mede a motivação intrínseca das pessoas mediante a aquisição do conhecimento. (AllaboutUX, 2016). A escala utilizada para este método não foi disponibilizada.

Rating Scale Mental Effort (RSME) - Escala de Esforço Mental: Desenvolvido pelo Holandês Zijlstra, esta escala indica 0 a $150 \mathrm{~mm}$ e ajuda a determinar quanto esforço mental foi necessário para concluir uma tarefa. (WAARD, D, 2016).

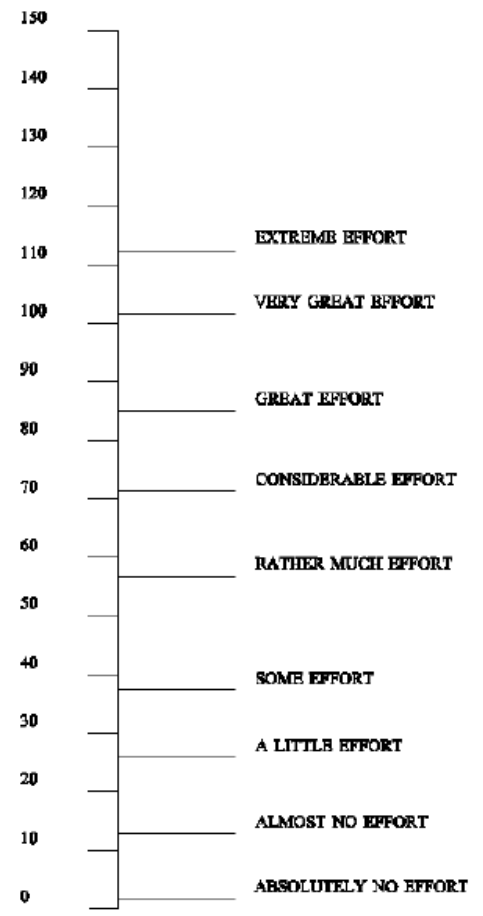

Figura 8: Exemplo da escala Rating Scale Mental Effort.

Fonte: (WAARD, D, 2016). 
Self Assessment Scale (SAM) - Escala de Auto Avaliação: método de avaliação pictórica não verbal que mede diretamente o prazer, alerta e dominância associada ao afeto de uma pessoa através de reações a uma grande variedade de estímulos. (BRADLEY, 1995).

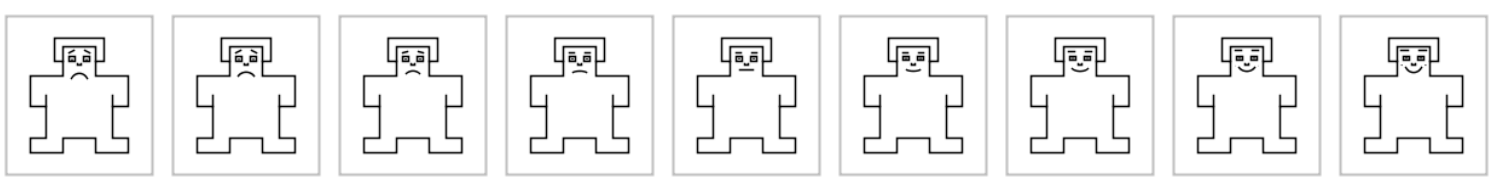

Figura 9: Exemplo da escala Self Assessment Scale. Fonte: (BRADLEY, 1995).

ServUX Questionnaire - sem sugestão para tradução: ferramenta projetada para avaliar a experiência do usuário em serviços oferecidos via web. (VäänänenVainio-Mattila; Segerstahl, 2009). A escala utilizada para este método não foi disponibilizada.

Software Usability Measurement Inventory (SUMI) - Inventário Medidor da Usabilidade Software: recomendado para qualquer organização que deseje medir a qualidade percebida de uso de software. (SUMI, 2016).

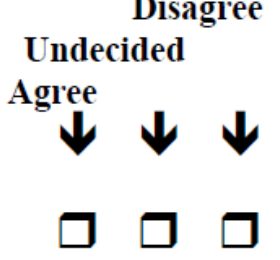

Figura 10: Exemplo da escala Software Usability Measurement Inventory. Fonte: (SUMI, 2016).

Website Analysis and Measurement Inventory (WAMMI) - Inventário de Análise e Medição para Website: método de análise da experiência do usuário no ambiente da Web. (WAMMI, 2016).

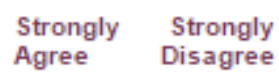

Figura 11: Exemplo da escala Website Analysis and Measurement Inventory. Fonte: (WAMMI, 2016).

\section{MATERIAIS E MÉTODOS}

Mediante o artigo de Veermereen et al. (2010), o qual os autores descrevem uma coletânea de 96 procedimentos de análise da experiência do usuário, foram selecionados os métodos categorizados no formato de questionários.

Uma pesquisa bibliográfica foi realizada com os 20 métodos desta categoria para compor suas definições e imagens das escalas exemplificadas. 
A busca obteve o retorno de 11 diferentes tipos escalas, as quais foram comparadas e as considerações descritas na Análise dos Resultados.

\section{ANÁLISE DOS RESULTADOS}

Das 11 escalas pesquisadas, é possível distinguir que 2 delas foram desenvolvidas em 2 eixos ( $x$ e y) e 9 delas em somente um eixo, sendo que dessas 9 , somente 1 se apresenta no eixo y e as demais no eixo $x$.

Aquelas que se utilizam de 2 eixos para sua construção possuem uma área maior para o detalhamento da emoção a ser investigada e ambas adotam a mesma posição para caracterizar o estado emocional que o usuário se encontra (agradável: lado direito, desagradável: lado esquerdo).

Todas as outras 9 escalas são delimitadas por níveis impares, sugerindo assim um estado neutro da análise: 1 com 3 níveis, 3 com 5 níveis, 2 com 7 níveis, 1 com 9 níveis e 1 com 15. Somente 1 escala o lado esquerdo está caracterizado como positivo, as outras o lado esquerdo é considerado como negativo.

\section{REFERÊNCIAS}

Associação Brasileira de Normas Técnicas ABNT NBR ISO 9241-210:2011. Ergonomia da interação humano-sistema. Parte 210: Projeto centrado no ser humano para sistemas interativos, 2011.

Affective Sciences: Disponível em: < http://www.affectivesciences.org/researchmaterial >. Acesso em 28 fev. 2016.

Allaboutux. Disponível em: < http://www.allaboutux.org/attrakdiff >. Acesso em 28 fev. 2016.

AttrakDiff. Disponível em: < http://attrakdiff.de/>. Acesso em 1 mar. 2016.

BRADLEY, M. M.; LANG, P. J. Measuring Emotion: The Self-Assessment Manikin and the Semantic Differencial. Journal of Behavior Therapy and Experimental Psychiatry, $v$. 25, n. 1, p. 49-59, 1994.

Game Experience Lab. Disponível em: < http://www.gamexplab.nl/index.php?page=home >. Acesso em 29 fev. 2016.

GERBING, D. W.; ANDERSON, J. C. An Updated Paradigm for Scale Development Incorporating Unidimensionality and Its Assessment. Journal of Marketing Research, v. 25, n. 2, p. 186-192, mai. 1995.

LAVIE, Talia; TRACTINSKY, N. Assessing dimensions of perceived visual aesthetics of web sites. International Journal of Human-Computer Studies, v. 60, n. 3, p. 269-298, mar. 2004.

LAWSON, B. How Designers Think: The Design Process Demystified. Great Britain. 4. ed., 2006.

NORMAN, D. Don Norman: depoimento [ago. 2008]. Entrevistador: Peter Merholz. San Francisco, Estados Unidos: UX Week 2008. Disponível em:

<https://vimeo.com/2963837>. Acesso em: 1 fev. 2016. 
RUSSELL, J. A.; WEISS, A.; MENDELSOHN, G. A. Affect grid: A single-item scale of pleasure and arousal. Journal of Personality and Social Psychology, v. 57, n. 3, p. 493502, 1989.

Self Determiation Theory. Disponível em: < http://selfdeterminationtheory.org/questionnaires/ >. Acesso em 29 fev. 2016.

SCHALL, A. The Future of UX Research: Uncovering the True Emotions of Our Users. The Magazine of the User Experience Professionals Association. 2015. Disponível em: < http://uxpamagazine.org/the-future-of-ux-research/ >. Acesso em 02 mar. 2016.

STAPPERS, P. J. Creative connections: user, designer, context, and tools. Personal and Ubiquitous Computing, v. 10, p. 95-100, 2006.

SUMI. Disponível em: < http://sumi.ucc.ie/ >. Acesso em 29 fev. 2016.

VÄÄTÄJÄ, H., KOPONEN, T.; ROTO, V. Developing practical tools for user experience evaluation: a case from mobile news journalism. In: European Conference on Cognitive Ergonomics: Designing beyond the Product - Understanding Activity and User Experience in Ubiquitous Environments, Proceedings... Finlandia, 2009.

VÄÄNÄNEN-VAINIO-MATTILA, K; SEGERSTAHL, K. A Tool for Evoluating Service User eXperience (ServUX): Development of a Modular Questionnaire. UX Thesis, 2009. Disponível em: < http://www.uxthesis.com/2009/a-tool-for-evaluating-service-userexperience-servux-development-of-a-modular-questionnaire/ >. Acesso em 29 fev. 2016.

VERMEEREN, A. P. O. S.; et al. User Experience Evaluation Methods: Current State and Development Needs. In: 6th Nordic Conference on Human-Computer Interaction, Iceland. Proceedings... Iceland: Reykjavik University, out. 2010. p. 521-530.

VOSS, K. E.; SPANGENBERG, E. R.; GROHMANN B. Measuring the Hedonic and Utilitarian Dimensions of Consumer Attitude. Journal of Marketing Research, v. 40, n. 3, p.310-320, ago. 2003.

WAARD, D. The Measurement of Drivers' Mental Workload. The Traffic Research Center VSC, University of Groningen: The Netherlands, 1996.

WAMMI. Disponível em: < http://www.wammi.com/index.html >. Acesso em 29 fev. 2016.

WATSON, D.; CLARK, L. A.; TELLEGAN, A. Development and Validation of Brief Measures of Positive and Negative Affect: The PANAS scales. Journal of Personality and Social Psychology, v. 54, n. 6, p.1063-1070, 1988.

WITMER, B. G.; SINGER, M. J. measuring presence in virtual environments: a presence questionnaire. Presence, v. 7, n. 3, p. 225-240, jun. 1998. 